\title{
Chronic Kidney Disease: Socioeconomic Impact. Findings from a Two Center Study in Southwestern Nigeria
}

\author{
Uduagbamen $\mathrm{PK}^{1,2}$, Ogunkoya $\mathrm{JO}^{3}$, Alalade $\mathrm{BA}^{4}$, Oyelese $\mathrm{AT}^{5}$, Nwogbe $\mathrm{IC}^{1}$, \\ Eigbe $\mathrm{SO}^{1}$, Timothy $\mathrm{OR}^{1}$
}

${ }^{1}$ Division of Nephrology and Hypertension, ${ }^{3}$ Pulmonology Unit, Department of Internal Medicine, Ben Carson (Snr) School of Medicine, Babcock University/ Babcock University Teaching Hospital, Ilishan-Remo, Nigeria ${ }^{2}$ Nephrology Unit, ${ }^{4}$ Diabetes and Metabolism Unit, Department of Internal Medicine, Federal Medical Centre, Abeokuta, Nigeria.

${ }^{5}$ Department of Hematology and Blood Transfusion, Ben Carson (Snr) School of Medicine, Babcock University/Babcock University Teaching Hospital, Ilishan-Remo, Nigeria

Corresponding Author: Peter Uduagbamen

\begin{abstract}
Introduction: Despite the rising prevalence of chronic kidney disease (CKD), access to adequate renal care is still not available to a very large part of the populace, essentially due to inadequate funds and this has further heightened the burden of the disease on patients and the general society. Measures are therefore needed to highlight this health challenge and proffer solutions.

Methods: A comparative study in which 354 consented participants with CKD stage 3-5 gave history, were examined and had blood taken for serum biochemistry and hematocrit to access kidney function.

Results: Two hundred and thirty six males and 118 females participated. The mean age of the participants was $52.11 \pm 6.04$ yrs. A greater percentage $(44.6 \%)$ of participants had hypertension as cause of CKD and earned a monthly income less than the national minimum wage (47.7\%). A greater proportion of participants had tertiary education $(51.4 \%)$, were married $(64.1 \%)$ and travelled less than 50 kilometers $(67.5 \%)$ to access renal care. The health insured were more likely to be males $(\mathrm{P}=0.002)$, aged $(\mathrm{P}<0.001)$ have higher hematocrit $(\mathrm{P}=0.002)$, albumin $(\mathrm{P}=0.06)$, bicarbonate $(\mathrm{P}=0.04)$ and GFR $(\mathrm{P}=0.01)$.

The health insured had more frequent dialysis $(\mathrm{P}<0.001)$ and erythropoietin use $(\mathrm{P}<0.001$. Forty percent of the health insured had renal transplant compared to $1.6 \%$ of the uninsured, $\mathrm{P}<0.001$.

The insured were more associated with IDHT as the uninsured were more associated with IDH. The health insured had a mean dialysis dose $(\mathrm{Kt} / \mathrm{V} 1.34 \pm 0.9)$ compared to $1.13 \pm 0.5$ for the uninsured, $\mathrm{P}<0.001$. The dialysis dose was positively correlated with frequency of dialysis $(\mathrm{P}<0.001)$, and erythropoietin $(\mathrm{P}<0.001)$ but was negatively correlated with age $(\mathrm{P}=0.01)$ and serum creatinine $(\mathrm{P}=0.004)$. Predictors of dialysis dose were insurance status, frequency of dialysis, and erythropoietin, hematocrit, serum albumin and bicarbonate.

Conclusion: Only $11.9 \%$ of the CKD cohorts had health insurance coverage and they were more likely to be males, aged, highly educated, with higher hematocrit, and albumin. The health insured had a mean dialysis dose of Kt/V $1.34 \pm 0.9$ as against $1.13 \pm 0.5$ for the uninsured. The uninsured had more metabolic acidosis, were younger and being the most active working population, their affectation only further worsens the burden associated with CKD.
\end{abstract}

Keywords: health insured, intradialysis hypotension, intradialysis hypertension, dialysis dose

\section{INTRODUCTION}

Chronic kidney disease (CKD) has become a global socioeconomic and health burden, more so in low income nations (LINs) and even more so in sub Sahara Africa (SSA). ${ }^{1}$ The prevalence of CKD 
worldwide is on the increase and the cost of treating an individual with CKD is expected to increase, with increased burden to patients, care givers and the governments, as the stage of the disease increased. ${ }^{2}$ Management in early (stages 1 and 2) CKD essentially involves prompt and effective management of etiologic factor, blood pressure control, prevention and control of proteinuria, as well as an effective counselling on dietary discretion, prevention/halting progression of atherosclerosis, and the prevention of thrombotic episodes and their complications. ${ }^{3}$ Treatment at these stages commonly involves measure with little or no cost implication or measures, some of which are affordable to the populace including most of the indigent individuals.

The third, fourth and the non-dialytic phase of stage 5 CKD present with most of the known disease features, involving constitutional symptoms, worsening blood pressure, hyperparathyroidism and CKD mineral bone disease (CKD-BMD), proteinuria, anemia, metabolic acidosis (MA) dyslipidemia and left ventricular hypertrophy $(\mathrm{LVH}){ }^{4}$ Treatment at this stages involves stricter control of $\mathrm{BP}$ and proteinuria, treatment, and control of hyperparathyroidism and bone disease and, anemia, dyslipidemia, reduction in protein intake amongst others. These stages of CKD are associated with a reduced quality of life (QOL) and increase risk of several complications, some of which could be fatal particularly cardiovascular events. ${ }^{5}$

The dialytic phase of stage 5 CKD (uremic phase) in most LINs, is unfortunately, the part in which patients commonly present for treatment. This is the phase of the disease where patients QOL are lowest, burden to care givers, governments and society are worse, cost of treatment highest and compliance levels are least. ${ }^{6}$ In addition to regimen used in managing earlier stages of CKD, the dialytic phase of stage 5 CKD commonly involves blood transfusions (less common in developed nations), use of intravenous erythropoiesis stimulating agents (ESAs), dialysis and renal transplant. ${ }^{1}$

In most LINs there is delayed referral of pre CKD conditions (hypertension, diabetes, sickle cell anemia and others) from the primary health care centers to physicians and nephrologists, and from physicians to nephrologists. ${ }^{7}$ Delay is also common in the preparation of many of these patients for renal replacement therapy (RRT) by the nephrologists in these settings due to socioeconomic, educational and cultural limitations. The kidney disease improving global outcome (KDIGO) recommended referral to nephrologist before stage 3 disease, even in LINs. ${ }^{8}$

Arije et $\mathrm{al}^{9}$ reported from Ibadan that not more than $30 \%$ of patients are able to continue dialysis treatment beyond 3 months. Likewise Akinsola et $\mathrm{al}^{10}$ had found that some patients had to travel for up to one hundred and forty kilometers to access dialysis treatment. Though many dialysis centers have been established in the last decade, they are still mostly concentrated in the urban centers. ${ }^{11}$

In Nigeria, apart from a very few wealthy, or privileged workers (or relatives) of a few multinational organizations, maintenance hemodialysis (MHD) and erythropoietin treatment is routinely given twice or less, even this is unaffordable to majority of the populace. ${ }^{12}$ Under-dialysis is expected to lead to inadequate dialysis, poor treatment outcome and quality of life (QOL), and premature death. ${ }^{13}$

In most LINs, like Nigeria, the rate of unemployment is very high and for many employed, under payment, delayed payment for services provided are very common. The national health insurance schemes (NHIS) of the Federal, and those of regional governments and the multinational corporations has about $3 \%$ of the population enrolled into the various schemes leaving more than $95 \%$ of the populace at the mercy of funds from self, families, religious bodies, philanthropists and friends. In Nigeria, the national minimum monthly wage is $\$ 60$. The cost of a session of 
hemodialysis (arguably the only RRT modality available to Nigerian adults with kidney disease) ranges from $\$ 60$ to $\$ 150$, the monthly (twelve sessions) cost range from $\$ 720$ to $\$ 1800$ while the monthly cost of erythropoietin (4000IU x 12 doses) is about $\$ 250$. This is in addition to the cost of antihypertensives, diuretics, antibiotics, hematinic, many other drugs and routine investigations. The socioeconomic outcome of CKD in LINs is further worsened by the demographics of CKD, where infective causes such as chronic glomerulonephritis (CGN) and chronic pyelonephritis (which are commoner in the actively working population) are among the leading causes of the disease. ${ }^{15}$

Studies highlighting poor treatment outcome in LINs like Nigeria have majorly dealt with specific causes of poor treatment outcome, but literature detailing the contribution of the various implicated factors, particularly socioeconomic, cultural and educational is however scares. This study assesses the contribution of socioeconomic, cultural and educational factors to the poor treatment outcome in Nigeria, a LIN, and ascertains their correlates with intradialysis events and dialysis dose.

\section{MATERIALS AND METHODS}

This was 30 months comparative study carried out at the nephrology and hypertension clinics, and dialysis suites of Federal Medical Centre, Abeokuta (JanuaryDecember 2017) and Babcock University Teaching Hospital, Ilishan-Remo, Nigeria, from August 2019 to January 2021. Recruitment into the study was by consecutive sampling in which three hundred and fifty four participants with CKD according to the KDOQI 2012 criteria $^{16}$ from stage $3-5$, that is, with glomerular filtration rate (GFR) $<60$ $\mathrm{ml} / \mathrm{min} / 1.73 \mathrm{~m}^{2}$ participated. Participants who had full health coverage (insurance), and those without full health insurance were grouped into two.
Exclusion criteria were: age less than 16 years, and participants with renal graft, pelvic tumors, infections or sessions less than 2 hours.

Variables taken from history, patients' case notes and dialysis notes included age, sex, level of educational attainment, sponsorship of treatment, estimated average monthly income, distance between participant's residence and treatment facility, preceding pharyngitis or skin sepsis, type and cause of CKD, months on MHD and number of antihypertensive drugs. Also entered were dialysis duration, blood flow rate (BFR), ultrafiltration volume (UFV), frequency of dialysis and erythropoietin use, intradialysis events and their timings, and post dialysis weight for preceding session. Also retrieved were doses of erythropoietin (4000 IU), intravenous Iron (200mg) and blood transfusion.

Participants' height and weight were measure according to standardized protocols. The oxygen saturation $\left(\mathrm{SPO}_{2}\right)$, pulse rate (PR), blood pressure (BP)] were measured after five minutes rest and documented. All BP readings were taken manually. The interdialytic weight gain (IDWG), the vital signs, the disease condition and participant's peculiarities like age and sex were used to formulate the dialysis prescription. For the non-dialyzing cohorts, blood sample was taken from a peripheral vein but for dialyzing cohorts, blood was taken from the dialysis catheters after withdrawing the sterilizing fluid and heparinized saline. Arterial and venous portals were flushed with heparinized saline and participants were collected through the arterial and then the venous portal. At the end of treatment time, dialysate flow (only) was stopped and the blood flow rate was reduced to $100 \mathrm{ml} / \mathrm{min}$. Five minutes after this, blood was taken from the arterial portal, first, for serum electrolyte (minimizes access recirculation) and then the HCT. ${ }^{17}$ The urea reduction ratio (URR) and $\mathrm{Kt} / \mathrm{V}$ (with Daugirdas second generation logarithmic estimation of single 
pool) were calculated ${ }^{18}$ Serum albumin was determined using the bromocresol green method. It overestimates albumin in hypoalbuminemia and kidney disease (including dialysis patients) by about $3.5 \mathrm{~g} / \mathrm{dl}^{19}$

Data analysis was with SPSS 22, continuous variables, presented as means with standard deviation, were compared using student t-test. Categorical variables, presented as proportion and percentages, were compared using Chi square test or Fisher's exact test. $\mathrm{P}<0.05$ was considered statistically significant. Multivariate regression analysis was used to determine predictors of dialysis dose. The Human Ethics Committee of Federal Medical Centre, Abeokuta and Babcock University Teaching Hospital, Ilishan-Remo (FMCA/470/HREC/03/2017, NHREC/08/102015 and BUHREC/733/19, NHREC/24/01/2018) approved this study.

\section{Definitions}

The diagnosis of the etiology of CKD was not by kidney biopsy.

Hypertension: Blood pressure >140/90 mmHg. ${ }^{20}$

Diabetes: fasting blood sugar (FBS) $>126$ $\mathrm{mg} / \mathrm{dl}$, previous diagnosis or receiving hypoglycemic agents. ${ }^{21}$

Anemia: Hematocrit $<33 \%{ }^{22}$

Hypoalbuminemia: serum albumin $<35 \mathrm{mg} / \mathrm{dL}^{23}$

eGFR (CKD-EPI)-ml/min $/ 1.73 \mathrm{~m}^{224}$

Hypertension associated CKD:

Hypertension that is complicated by kidney disease seen from late middle age upwards.

Chronic glomerulonephritis: Kidney disease complicated by hypertension seen in the young and early middle age, with or without history of pharyngitis and skin sepsis in the past.

Targeted weight loss (TWL): Predialysis weight plus volume of administered fluid minus UFV. ${ }^{25}$

IDH: Intradialysis fall in SBP of $\geq 20$ $\mathrm{mmHg}$ with symptoms but without nursing intervention. ${ }^{26}$

IDHT: Intradialysis rise in SBP $>10$ mmHg. ${ }^{27}$
Dialysis dose: Normal ( $\mathrm{Kt} / \mathrm{V} \geq 1.2$ and URR $\geq 65.0 \%)$, low $(\mathrm{Kt} / \mathrm{V}<1.2$ and URR $<65.0 \%)$.

\section{RESULTS}

Table 1: Socioeconomic, cultural and educational characteristics of participant

\begin{tabular}{|c|c|c|}
\hline Variables & $\begin{array}{l}\text { Frequency } \\
\mathrm{N}=354\end{array}$ & $\begin{array}{l}\text { Percentage } \\
(\%)\end{array}$ \\
\hline $\begin{array}{l}\text { Sex } \\
\text { Males } \\
\text { Females }\end{array}$ & $\begin{array}{l}236 \\
118 \\
\end{array}$ & $\begin{array}{l}66.7 \\
33.3 \\
\end{array}$ \\
\hline $\begin{array}{l}\text { Age, years } \\
16-39 \\
40-64 \\
>65\end{array}$ & $\begin{array}{l}93 \\
208 \\
53 \\
\end{array}$ & $\begin{array}{l}26.3 \\
58.7 \\
15.0 \\
\end{array}$ \\
\hline $\begin{array}{l}\text { Educational attainment } \\
\text { None } \\
\text { Primary } \\
\text { Secondary } \\
\text { Tertiary }\end{array}$ & $\begin{array}{l}7 \\
59 \\
137 \\
151\end{array}$ & $\begin{array}{l}2.0 \\
16.7 \\
38.7 \\
42.6\end{array}$ \\
\hline $\begin{array}{l}\text { Estimated total monthly income in } \\
\text { naira }(\$) \\
<\# 30000(<\$ 60) \\
\# 30000-99999(<\$ 60-\$ 199.9) \\
\# 100000-499999(\$ 200-\$ 999.9) \\
\# 500000-999999(\$ 1000-\$ 1999.9) \\
>\# 1000000(>\$ 2000)\end{array}$ & $\begin{array}{l}169 \\
119 \\
52 \\
11 \\
3\end{array}$ & $\begin{array}{l}47.7 \\
33.6 \\
14.7 \\
3.1 \\
0.9 \\
\end{array}$ \\
\hline $\begin{array}{l}\text { Sponsorship } \\
\text { Self } \\
\text { Family } \\
\text { Institution: Full } \\
\quad \text { : Partial } \\
\text { Religious/Friends/Philanthropists }\end{array}$ & $\begin{array}{l}94 \\
182 \\
42 \\
10 \\
26\end{array}$ & $\begin{array}{l}26.6 \\
51.4 \\
11.9 \\
2.8 \\
7.3\end{array}$ \\
\hline $\begin{array}{l}\text { Distance to dialysis center }(\mathrm{km}) \\
<20 \\
20-49 \\
50-99 \\
>100\end{array}$ & $\begin{array}{l}56 \\
183 \\
67 \\
48\end{array}$ & $\begin{array}{l}15.8 \\
51.7 \\
18.9 \\
13.6\end{array}$ \\
\hline $\begin{array}{l}\text { Family set up } \\
\text { Single } \\
\text { Married } \\
\text { Widow/Widower }\end{array}$ & $\begin{array}{l}78 \\
227 \\
49 \\
\end{array}$ & $\begin{array}{l}22.0 \\
64.1 \\
13.9 \\
\end{array}$ \\
\hline $\begin{array}{l}\text { Etiology of CKD } \\
\text { Hypertension } \\
\text { Chronic glomerulonephritis } \\
\text { Diabetes } \\
\text { Obstructive uropathy } \\
\text { Others }\end{array}$ & $\begin{array}{l}128 \\
98 \\
33 \\
18 \\
10\end{array}$ & $\begin{array}{l}44.6 \\
34.1 \\
11.5 \\
6.3 \\
3.5\end{array}$ \\
\hline $\begin{array}{l}\text { Stages of CKD } \\
3 \mathrm{a} \\
3 \mathrm{~b} \\
4 \\
5 \text { (non-dialytic) } \\
5 \text { (dialytic) }\end{array}$ & $\begin{array}{l}6 \\
7 \\
13 \\
41 \\
287\end{array}$ & $\begin{array}{l}1.7 \\
2.0 \\
3.7 \\
11.6 \\
81.0\end{array}$ \\
\hline
\end{tabular}

CKD-chronic kidney disease

Three hundred and fifty four (236 males and 118 females) participants were studied. The mean age of the participants was $52.11 \pm 6.04$ years $(58.22 \pm 16.13$ years for non dialytic, and $50.17 \pm 11.17$ years for the dialytic). Participants in stages 3, 4, non dialytic 5 and dialytic 5 were $12(3.39 \%), 14$ (3.96\%), 41 (11.58\%) and $287(81.07 \%)$. A greater percentage $(44.6 \%)$ of participants had hypertension as cause of CKD and, had 
monthly income less than the national minimum wage $(47.7 \%)$. A greater percentage had tertiary education $(51.4 \%)$, were married $(64.1 \%)$ and travelled less than 50 kilometers $(67.5 \%)$ to access renal care.

The BP, creatinine and anion gap were significantly lower in the health insured compared to the uninsured, $\mathrm{P}<0.001, \quad \mathrm{P}<0.001, \quad \mathrm{P}<0.001$ respectively (Table 2). The serum bicarbonate, GFR, albumin and hematocrit were significantly higher in the health insured compared to the uninsured, $(\mathrm{P}=0.001),(\mathrm{P}<0.001),(\mathrm{P}=0.03)$ and $(\mathrm{P}=0.02)$ respectively.

\begin{tabular}{|c|c|c|c|c|}
\hline Variables & $\begin{array}{l}\text { All participants } \\
\mathrm{N}=354(\%)\end{array}$ & $\begin{array}{l}\text { Non-dialyzed CKD } \\
\mathrm{N}=67(\%)\end{array}$ & $\begin{array}{l}\text { Dialyzed CKD } \\
\mathrm{N}=287(\%)\end{array}$ & P-value \\
\hline $\begin{array}{l}\text { Sex } \\
\text { Males } \\
\text { Females }\end{array}$ & $\begin{array}{l}236(66.7) \\
118(33.3) \\
\end{array}$ & $\begin{array}{l}44(65.7) \\
23(34.3) \\
\end{array}$ & $\begin{array}{l}192(66.9) \\
95(33.1)\end{array}$ & 0.7 \\
\hline $\begin{array}{l}\text { Age, years } \\
16-39 \\
40-64 \\
\geq 65 \\
\end{array}$ & $\begin{array}{l}93(26.3) \\
208(58.7) \\
53(15.0)\end{array}$ & $\begin{array}{l}19(28.4) \\
37(55.2) \\
11(16.4)\end{array}$ & $\begin{array}{l}74(25.8) \\
171(59.6) \\
42(14.6) \\
\end{array}$ & 0.5 \\
\hline $\begin{array}{l}\text { Educational attainment } \\
\text { None } \\
\text { Primary } \\
\text { Secondary } \\
\text { Tertiary }\end{array}$ & $\begin{array}{l}7(2.0) \\
59(16.7) \\
137(38.7) \\
151(42.6)\end{array}$ & $\begin{array}{l}3(4.5) \\
15(22.4) \\
24(35.8) \\
25(37.3)\end{array}$ & $\begin{array}{l}4(1.4) \\
44(15.3) \\
113(39.4) \\
126(43.9)\end{array}$ & 0.04 \\
\hline BMI, $\mathrm{kg} / \mathrm{m}^{2}$ & $24.8 \pm 5.6$ & $25.8 \quad 11.4$ & $24.6 \pm 9.3$ & 0.06 \\
\hline Systolic BP (mean) mmHg & $160.0 \pm 16.8$ & $144.2 \pm 24.9$ & $163.7 \pm 14.7$ & $<0.001$ \\
\hline Diastolic BP (mean), mmHg & $95.5 \pm 9.9$ & $82.4 \pm 13.1$ & $98.5 \pm 8.9$ & $<0.001$ \\
\hline Serum sodium (mean) mmol/L & $130.1 \pm 7.9$ & $132.8 \pm 8.5$ & $129.5 \pm 8.2$ & 0.06 \\
\hline Serum potassium (mean) $\mathrm{mmol} / \mathrm{L}$ & $5.7 \pm 1.1$ & $5.5 \pm 1.3$ & $5.7 \pm 1.3$ & 0.8 \\
\hline Serum chloride (mean) mmol/L & $97.9 \pm 10.4$ & $98.5 \pm 6.6$ & $97.8 \pm 13.4$ & 0.1 \\
\hline Serum bicarbonate (mean) $\mathrm{mmol} / \mathrm{L}$ & $19.1 \pm 4.9$ & $21.5 \pm 5.7$ & $18.6 \pm 4.3$ & 0.001 \\
\hline Serum calcium (mean) mmol/L & $2.1 \pm 0.9$ & $2.0 \pm 0.4$ & $2.1 \pm 1.1$ & 0.05 \\
\hline Serum phosphate (mean) mmol/L & $2.2 \pm 1.5$ & $2.4 \pm 1.4$ & $2.2 \pm 1.3$ & 0.1 \\
\hline Serum urea (mean) $\mathrm{mmol} / \mathrm{L}$ & $18.8 \pm 9.7$ & $24.5 \pm 11.3$ & $17.5 \pm 6.3$ & $<0.001$ \\
\hline Serum creatinine (mean) umol/L & $749.5 \pm 33.5$ & $248.8 \pm .42 .8$ & $866.4 \pm 16.2$ & $<0.001$ \\
\hline Glomerular filtration rate, $\mathrm{ml} / \mathrm{min}$ & $9.7 \pm 3.6$ & $22.5 \pm 5.7$ & $6.7 \pm 1.4$ & $<0.001$ \\
\hline Serum albumin, $\mathrm{mg} / \mathrm{dL}$ & $31.6 \pm 7.9$ & $34.8 \pm 11.6$ & $30.9 \pm 7.4$ & 0.03 \\
\hline Hematocrit, \% & $24.7 \pm 4.9$ & $27.3 \pm 5.6$ & $24.1 \pm 5.8$ & 0.02 \\
\hline Mean anion gap, $\mathrm{mEq}$ & $24.6 \pm 5.5$ & $15.7 \pm 8.4$ & $26.7 \pm 8.7$ & $<0.001$ \\
\hline
\end{tabular}

CKD-chronic kidney disease, BMI-body mass index, BP-blood pressure

Table 3: Socioeconomic impact on clinical and laboratory profile of the pre dialytic population

\begin{tabular}{|c|c|c|c|c|}
\hline Variables & All participants $\mathrm{N}=67(\%)$ & Insured $\mathrm{N}=12(\%)$ & Uninsured $\mathrm{N}=55(\%)$ & P-value \\
\hline $\begin{array}{l}\text { Sex } \\
\text { Males } \\
\text { Females }\end{array}$ & $\begin{array}{l}44(65.7) \\
23(34.3)\end{array}$ & $\begin{array}{l}9(75.0) \\
3(25.0)\end{array}$ & $\begin{array}{l}35(63.6) \\
20(36.4)\end{array}$ & 0.002 \\
\hline $\begin{array}{l}\text { Age, years } \\
16-39 \\
40-64 \\
>65 \\
\end{array}$ & $\begin{array}{l}19(28.4) \\
37(55.2) \\
11(16.4) \\
\end{array}$ & $\begin{array}{l}1(8.3) \\
4(33.3) \\
7(58.4) \\
\end{array}$ & $\begin{array}{l}18(32.7) \\
33(60.0) \\
4(7.3) \\
\end{array}$ & $<0.001$ \\
\hline $\begin{array}{l}\text { Erythropoietin use } \\
\text { No } \\
\text { Yes }\end{array}$ & $\begin{array}{l}54(80.6) \\
13(19.4)\end{array}$ & $\begin{array}{l}6(50.0) \\
6(50.0)\end{array}$ & $\begin{array}{l}48(87.3) \\
7(12.7)\end{array}$ & $<0.001$ \\
\hline Mean BMI, kg/m ${ }^{2}$ & $25.8 \pm 11.4$ & $27.4 \pm 11.6$ & $25.5 \pm 12.4$ & 0.03 \\
\hline Mean systolic BP & $144.2 \pm 24.9$ & $143.1 \pm 16.8$ & $144.4 \pm 18.8$ & 0.1 \\
\hline Mean diastolic BP & $82.4 \pm 13.1$ & $80.9 \pm 10.6$ & $82.7 \pm 10.7$ & 0.05 \\
\hline Mean hematocrit & $27.3 \pm 5.6$ & $29.0 \pm 6.3$ & $26.9 \pm 5.5$ & 0.002 \\
\hline Mean albumin & $34.8 \pm 11.6$ & $35.8 \pm 7.9$ & $34.6 \pm .9$ & 0.6 \\
\hline Mean sodium & $132.8 \pm 8.5$ & $134.6 \pm 12.9$ & $132.4 \pm 9.3$ & 0.05 \\
\hline Mean potassium & $4.6 \pm 1.8$ & $4.7 \pm 2.0$ & $4.6 \pm 1.5$ & 0.07 \\
\hline Mean bicarbonate & $21.5 \pm 5.7$ & $22.0 \pm 7.0$ & $21.4 \pm 4.7$ & 0.04 \\
\hline Mean chloride & $95.5 \pm 6.6$ & $98.6 \pm 8.1$ & $94.8 \pm 10.2$ & 0.03 \\
\hline Mean anion gap & $15.7 \pm 8.4$ & $15.0 \pm 6.4$ & $15.9 \pm 8.7$ & 0.05 \\
\hline Mean calcium & $2.0 \pm 0.4$ & $2.2 \pm 1.6$ & $1.9 \pm 0.6$ & 0.001 \\
\hline Mean phosphate & $2.4 \pm 1.4$ & $2.4 \pm 1.5$ & $2.4 \pm 1.1$ & 0.5 \\
\hline Mean creatinine & $248.8 \pm .42 .8$ & $195.4 \pm 36.9$ & $260.4 \pm 39.5$ & $<0.001$ \\
\hline Mean GFR & $22.6 \pm 6.2$ & $25.9 \pm 8.6$ & $21.9 \pm 9.8$ & 0.01 \\
\hline
\end{tabular}

BMI-body mass index, BP-blood pressure, GFR-glomerular filtration rate 
In the predialytic population (Table 3 ), the health insured were more likely to be males $(\mathrm{P}=0.002)$, aged $(\mathrm{P}<0.001)$, lower blood pressure, higher hematocrit $(\mathrm{P}=0.002)$, albumin $(\mathrm{P}=0.06)$, bicarbonate $(\mathrm{P}=0.04)$ with lower anion gap $(\mathrm{P}-0.05)$ and higher GFR ( $\mathrm{P}=0.01)$.

In the dialytic population (Table 4), the insured participants were more likely to be males $(\mathrm{P}=0.04)$, and associated with aging $(\mathrm{P}=0.03)$, frequent dialysis $(\mathrm{P}<0.001)$ and erythropoietin use $(\mathrm{P}<0.001)$, lower blood pressure, and higher hematocrit $(\mathrm{P}=0.03)$, albumin $(\mathrm{P}=0.04)$ and GFR $(\mathrm{P}=0.04)$. Forty percent of the insured participants went on to have renal transplant compared to $1.6 \%$ of the uninsured participants, $\mathrm{P}<0.001$.

\begin{tabular}{|c|c|c|c|c|}
\hline Variables & $\begin{array}{l}\text { All participants } \\
287(\%)\end{array}$ & $\begin{array}{l}\text { Insured } \\
\mathbf{N}=30(\%)\end{array}$ & $\begin{array}{l}\text { Uninsured } \\
\mathrm{N}=257(\%)\end{array}$ & P-value \\
\hline $\begin{array}{l}\text { Sex } \\
\text { Males } \\
\text { Females } \\
\end{array}$ & $\begin{array}{l}192(66.9) \\
95(33.1) \\
\end{array}$ & $\begin{array}{l}22(73.3) \\
8(26.7) \\
\end{array}$ & $\begin{array}{l}170(66.1) \\
87(33.9) \\
\end{array}$ & 0.04 \\
\hline $\begin{array}{l}\text { Age, yrs } \\
16-39 \\
40-64 \\
>65\end{array}$ & $\begin{array}{l}83(28.9) \\
152(53.0) \\
52(18.1) \\
\end{array}$ & $\begin{array}{l}3(10.0) \\
19(63.3) \\
8(26.7)\end{array}$ & $\begin{array}{l}80(31.1) \\
133(51.8) \\
44(17.1)\end{array}$ & 0.03 \\
\hline $\begin{array}{l}\text { Dialysis sessions/week } \\
1 \\
2 \\
3\end{array}$ & $\begin{array}{l}99(34.5) \\
143(49.8) \\
45(15.7)\end{array}$ & $\begin{array}{l}0(0.0) \\
2(6.7) \\
28(93.3)\end{array}$ & $\begin{array}{l}99(38.5) \\
141(54.9) \\
17(6.6) \\
\end{array}$ & $<0.001$ \\
\hline $\begin{array}{l}\text { Erythropoietin use } \\
1 \\
2 \\
3 \\
\end{array}$ & $\begin{array}{l}87(30.3) \\
152(53.0) \\
48(16.7) \\
\end{array}$ & $\begin{array}{l}1(3.3) \\
8(26.7) \\
21(70.0)\end{array}$ & $\begin{array}{l}86(33.5) \\
144(56.0) \\
27(10.5) \\
\end{array}$ & $<0.001$ \\
\hline Mean BMI, kg/m² & $24.6 \pm 9.3$ & $26.1 \pm 11.6$ & $24.4 \pm 9.7$ & 0.05 \\
\hline Mean systolic BP & $164.7 \pm 14.7$ & $164.6 \pm 10.8$ & $164.8 \pm 11.7$ & 0.7 \\
\hline Mean diastolic BP & $98.5 \pm 8.9$ & $96.7 \pm 8.3$ & $98.7 \pm 3$ & 0.06 \\
\hline Mean hematocrit & $24.1 \pm 5.8$ & $27.2 \pm 6.6$ & $23.7 \pm 9$ & 0.03 \\
\hline Mean albumin & $30.9 \pm 7.4$ & $33.5 \pm 8.4$ & $30.6 \pm 4.7$ & 0.04 \\
\hline Mean sodium & $129.5 \pm 8.2$ & $132.9 \pm 9.6$ & $129.1 \pm 12.7$ & 0.07 \\
\hline Mean potassium & $5.7 \pm 1.3$ & $5.1 \pm 0.9$ & $5.7 \pm 1.2$ & 0.05 \\
\hline Mean bicarbonate & $18.6 \pm 4.3$ & $20.7 \pm 7.1$ & $18.4 \pm 5.5$ & 0.04 \\
\hline Mean chloride & $97.8 \pm 13.4$ & $99.6 \pm 10.8$ & $97.6 \pm 14.2$ & 0.06 \\
\hline Mean anion gap & $26.7 \pm 8.7$ & $19.3 \pm 4.6$ & $27.6 \pm 7.2$ & 0.004 \\
\hline Mean calcium & $2.1 \pm 1.1$ & $2.2 \pm 1.4$ & $2.1 \pm 0.6$ & 0.1 \\
\hline Mean phosphate & $2.2 \pm 1.3$ & $2.3 \pm 1.1$ & $2.2 \pm 0.8$ & 0.3 \\
\hline Mean creatinine & $866.4 \pm 16.2$ & $812.3 \pm$ & $872.8 \pm 21.7$ & 0.002 \\
\hline Mean GFR & $6.7 \pm 1.4$ & $8.0 \pm 1.7$ & $6.5 \pm 2,0$ & 0.04 \\
\hline $\begin{array}{l}\text { Kidney transplant } \\
\text { No } \\
\text { Yes }\end{array}$ & $\begin{array}{l}271(94.4) \\
16(5.6)\end{array}$ & $\begin{array}{l}18(60.0) \\
12(40.0)\end{array}$ & $\begin{array}{l}253(98.4) \\
4(1.6)\end{array}$ & $<0.001$ \\
\hline
\end{tabular}

\begin{tabular}{l} 
Table 5: Intradialysis events in the dialytic population \\
\begin{tabular}{|l|l|l|l|l|}
\hline Variables & $\begin{array}{l}\text { All sessions } \\
\mathbf{N}=\mathbf{1 6 8 8}(\boldsymbol{\%})\end{array}$ & $\begin{array}{l}\text { Insured } \\
\mathbf{N}=\mathbf{1 8 0}(\boldsymbol{\%})\end{array}$ & $\begin{array}{l}\text { Uninsured } \\
\mathbf{N = 1 5 0 8}(\boldsymbol{\%})\end{array}$ & P-value \\
\hline IDH & $335(19.8)$ & $6(3.3)$ & $329(21.8)$ & 0.001 \\
\hline Insignificant BP change & $952(56.4)$ & $97(53.9)$ & $855(56.7)$ & 0.05 \\
\hline IDHT & $401(23.8)$ & $77(42.8)$ & $324(21.5)$ & 0.03 \\
\hline
\end{tabular} \\
\hline
\end{tabular}

The episodes of IDH (19.8\%) were fewer than IDHT (23.8\%). The insured were more likely to experience IDHT than IDH (Table 5). The uninsured participants were more likely to experience IDH than IDHT.

The mean dialysis dose for the study was $1.16 \pm 0.4$ (insured $1.34 \pm 0.9$, uninsured $1.13 \pm 0.5$ ) (males $1.21 \pm 0.3$, and females $1.07 \pm 0.5$ ). The mean dialysis dose was adequate, low and very low in $15.2 \%$, $48.9 \%$ and $35.9 \%$ sessions respectively. The dialysis dose was higher in males than females, $\mathrm{P}=0.04$, (Table 6). The dialysis dose was positively correlated with health insurance $(\mathrm{P}<0.001)$, frequency of dialysis $(\mathrm{P}<0.001)$, and erythropoietin $(\mathrm{P}<0.001)$, bicarbonate $\quad(\mathrm{P}<0.001), \quad$ hematocrit $(\mathrm{P}<0.001)$ and albumin $(\mathrm{P}<0.001)$, and was 
negatively correlated with age $(\mathrm{P}=0.01), \quad(\mathrm{P}<0.001)$. creatinine $(\mathrm{P}=0.004)$, and potassium

Table 6: Relationship between the dialysis dose and variables of the dialytic population.

\begin{tabular}{|c|c|c|c|c|c|}
\hline Variables & $\begin{array}{l}\mathrm{Kt} / \mathrm{V}<1.2 \\
\mathrm{~N}=1432(\%)\end{array}$ & $\begin{array}{l}\mathrm{Kt} / \mathrm{V}>1.2 \\
\mathrm{~N}=256(\%)\end{array}$ & OR & $95 \% \mathrm{CI}$ & P-value \\
\hline $\begin{array}{l}\text { Sex } \\
\text { Males } \\
\text { Females }\end{array}$ & $\begin{array}{l}941(83.7) \\
491(87.1)\end{array}$ & $\begin{array}{l}183(16.3) \\
73(12.9)\end{array}$ & 1.23 & $1.04-2.57$ & 0.04 \\
\hline $\begin{array}{l}\text { Age, yrs } \\
<65 \\
>65\end{array}$ & $\begin{array}{l}1133(82.3) \\
299(96.1)\end{array}$ & $\begin{array}{l}244(17.7) \\
12(3.9)\end{array}$ & 2.86 & $1.15-4.82$ & 0.01 \\
\hline $\begin{array}{l}\text { Insurance } \\
\text { No } \\
\text { Yes }\end{array}$ & $\begin{array}{l}1375(96.7) \\
57(21.4)\end{array}$ & $\begin{array}{l}47(3.3) \\
209(78.6)\end{array}$ & 7.33 & $4.42-10.74$ & $<0.001$ \\
\hline $\begin{array}{l}\text { Dialysis sessions/week } \\
<3 \\
3 \\
\end{array}$ & $\begin{array}{l}1256(96.5) \\
176(45.5)\end{array}$ & $\begin{array}{l}45(3.5) \\
211(54.5)\end{array}$ & 4.48 & $4.29-7.36$ & $<0.001$ \\
\hline $\begin{array}{l}\text { Erythropoietin use } \\
<3 \\
3\end{array}$ & $\begin{array}{l}1300(97.5) \\
132(37.3)\end{array}$ & $\begin{array}{l}34(2.5) \\
222(62.7)\end{array}$ & 5.36 & $3.82-8.26$ & $<0.001$ \\
\hline $\begin{array}{l}\text { Etiology of CKD } \\
\text { Hypertension } \\
\text { Chronic glomerulonephritis } \\
\text { Others }\end{array}$ & $\begin{array}{l}623(82.5) \\
489(83.0) \\
320(93.0)\end{array}$ & $\begin{array}{l}132(17.5) \\
100(17.0) \\
24(7.0)\end{array}$ & $\begin{array}{l}1 \\
1.26 \\
3.86\end{array}$ & $\begin{array}{l}0.62-2.05 \\
0.96-13.59\end{array}$ & 0.03 \\
\hline $\begin{array}{l}\text { Systolic BP, mmHg } \\
<140 \\
>140\end{array}$ & $\begin{array}{l}270(81.3) \\
1162(85.7)\end{array}$ & $\begin{array}{l}62(18.7) \\
194(14.3)\end{array}$ & 1.15 & $0.65-1.82$ & 0.05 \\
\hline $\begin{array}{l}\text { Hematocrit, \% } \\
<33 \\
>33\end{array}$ & $\begin{array}{l}1342(90.6) \\
90(43.9)\end{array}$ & $\begin{array}{l}141(9.4) \\
115(56.1)\end{array}$ & 4.28 & $2.9-7.16$ & $<0.001$ \\
\hline $\begin{array}{l}\text { Albumin, } \mathrm{mg} / \mathrm{dL} \\
<35 \\
>35\end{array}$ & $\begin{array}{l}1331(91.6) \\
101(43.0)\end{array}$ & $\begin{array}{l}122(8.4) \\
134(57.0)\end{array}$ & 5.12 & $2.96-8.15$ & $<0.001$ \\
\hline $\begin{array}{l}\text { Potassium, } \mathrm{mmol} / \mathrm{L} \\
<5.5 \\
>5.5\end{array}$ & $\begin{array}{l}185(53.0) \\
1247(93.1)\end{array}$ & $\begin{array}{l}164(47.0) \\
92(6.9)\end{array}$ & 4.89 & $2.46-6.94$ & $<0.001$ \\
\hline $\begin{array}{l}\text { Bicarbonate, mmolL } \\
<22 \\
>22\end{array}$ & $\begin{array}{l}1419(97.2) \\
13(5.7)\end{array}$ & $\begin{array}{l}41(2.8) \\
215(94.3)\end{array}$ & 8.79 & $5.11-12.98$ & $<0.001$ \\
\hline $\begin{array}{l}\text { Creatinine, umol/L } \\
<500 \\
>500\end{array}$ & $\begin{array}{l}752(77.0) \\
680(95.5)\end{array}$ & $\begin{array}{l}224(23.0) \\
32(4.5)\end{array}$ & 3.24 & $2.96-5.03$ & 0.004 \\
\hline
\end{tabular}

OR-odds ratio, CI-confidence interval, CKD-chronic kidney disease, BP-blood pressure

From the univariate analysis, variables with $\mathrm{P}<0.025$ were enter into the multivariate model (Table 7) from where health insurance, frequency of dialysis, and erythropoietin, hematocrit, serum albumin, bicarbonate and creatinine predicted dialysis dose.

Table 7: Multivariate regression analysis

\begin{tabular}{|l|l|l|l|}
\hline Tariables & aOR & $\mathbf{9 5 \%}$ CI & P-value \\
\hline Age & 1.02 & $0.85-1.92$ & 0.06 \\
\hline Full sponsorship & 6.89 & $5.38-13.54$ & $<0.001$ \\
\hline Frequency of dialysis & 3.52 & $1.78-4.45$ & 0.001 \\
\hline Frequency of erythropoietin & 3.61 & $2.55-6.27$ & 0.001 \\
\hline Hematocrit & 4.85 & $3.66-7.93$ & $<0.001$ \\
\hline Serum albumin & 3.13 & $1.72-4.06$ & 0.002 \\
\hline Serum potassium & 1.25 & $1.52-2.89$ & 0.05 \\
\hline Serum bicarbonate & 6.97 & $4.82-12.94$ & $<0.001$ \\
\hline Serum creatinine & 1.78 & $0.99-2.27$ & 0.03 \\
\hline
\end{tabular}

aOR-adjusted odds ratio, CI-confidence interval

\section{DISCUSSION}

We found that only $11.9 \%$ of the CKD cohorts $(17.9 \%$ in the predialytic phase and $10.5 \%$ in the dialytic phase) had full access to renal care. This leaves a very large majority $(88.1 \%)$ without full access. Nearly half $(47.7 \%)$ of the CKD cohorts were earning less than the monthly national minimum wage of 60 USD. The fully sponsored were more likely to have IDHT than IDH as the non-sponsored were more likely to have IDH than IDHT. This findings only but confirms the $3 \%$ population coverage by the Nigeria "Nation Health Insurance Scheme" (NHIS). ${ }^{14}$ The health insurance coverage is rather very low compared to other developing nations in South America like Colombia, and India in Asia. In Indian the Federal and States 
government with private insurance schemes cover about $25 \%$ of the population. Another disheartening truth is the known fact that CKD is more prevalent among the poor and rural dwellers, this disadvantageous position is further compounded by the fact that most of the renal and dialysis centers are located in the urban centers. ${ }^{11,} 28$ An addition to these challenges is the reported long distances patients had to travel to access dialysis as was found in this study, considering the functional states of most of the roads, particularly the rural roads. ${ }^{10}$

The insured participants had higher BMI, lower blood pressure, higher hematocrit and albumin than the uninsured, factors that that have been reported to contribute separately, and in synergy, to a higher dialysis dose, better QOL, reduce morbidity and mortality. The benefits of health insurance are further displayed as these patient progresses in the disease to the extent of requiring renal transplant. The insured in this study could be liken to citizens of the developed nations who enjoy better access to the health care provided by their governments, enabling them to access, uninterruptedly, even the most expensive investigations and treatment regimen require for managing $\mathrm{CKD}^{29}$

In this study, the insured were more likely to be aged compared to the uninsured, again, this mimics the older CKD population in developed nations who developed CKD commonly from chronic non communicable, and degenerative diseases unlike in Nigeria and many low income nations where infection related etiological factors are still very common. ${ }^{15}$ ${ }^{30}$ The higher male participation, and their higher enrolment rate into the treatment program of the insurance schemes in this study is in agreement with earlier studies in Nigeria and many nations in $\mathrm{SSA}^{31-32}$ The cultural background in Nigeria and many African nations favor males who are therefore more educated, favored to secure employments opportunities and tend to seek health care earlier than females. ${ }^{33}$ The higher insurance rate for males in this study further adds credence to the reported socioeconomic and cultural bias against women. The activation of the sympathoadrenergic system by the use of sympathomimetic agents is reported to be more in males who also show lesser response to sympathetic inhibition with inhibitors of the renin angiotensin aldosterone system (RAAS), ${ }^{34}$ and this in addition to the anti-apoptotic properties of testosterone in the renal tubules partly explains the higher prevalence of CKD in males, and also justifies the faster CKD progression rate in them. ${ }^{35}$ The higher proportion of males in the dialytic compared to the predialytic phase in this study is in agreement with these earlier findings. ${ }^{34,35}$

The higher BMI of the insured in this study reflects their better nutritional intake more so considering the fact that they also presented with lower blood pressure, thereby ruling out the possibility of higher BMI that is secondary to fluid retention, a feature that is not uncommon in CKD. ${ }^{36}$

Despite the lower BP of the insured, they were more likely to develop intradialysis hypertension than IDH. Their more frequent dialysis sessions entails lower concentrations of nitrogenous waste and retained fluid compared to the uninsured, as was found in our study. During dialysis, the lower osmotic gradient across the dialyzer membrane is associated with lesser osmotic shift between the blood and dialyzer compartments. The lower fluid shift seen in this case entails lower ultrafiltration volume (UFV) and this is associated with lesser blood pressure reductions hence intradialysis hypotension are less commonly experienced in these sessions compared to IDHT. ${ }^{37}$ The better blood pressure control in them may attenuate the cardiovascular damage hence even with larger UFV, they are more likely to mount a compensatory activation of the sympatheto-adrenergic system (in response to ultrafiltration) that may return the BP to predialysis levels or even overshoot it to cause IDHT. ${ }^{37}$

The better biochemical profile of the insured in comparison to the uninsured in 
our study, reflects the overall benefits of full access to renal care from the screening through the investigations, treatment and follow up of patients. These benefits cover a wide range of associations relating diseases and clinical outcome. The lower prevalence of metabolic acidosis in them helps in mitigating the occurrence and symptomatology of CKD-mineral bone disease. $^{38}$ Chronic metabolic acidosis accelerates the aging process ${ }^{39}$ clinically therefore, the insured is more likely to exhibit greater vigor and this, in the working class age group, could mitigate the known features of the disease, improve productivity, lessen the burden on care givers, families and governments. The greater access to erythropoiesis stimulating agents (ESAs) reduces the need for transfusion, and the attendant benefit of improved compatibility and reduced need for desensitization and plasmapheresis prior to transplant.

The higher dialysis dose in the insured is similar to what is obtained in the developed nations and this represents the contribution of the various segments of management and their protocol. ${ }^{38}$ While the $15.2 \%$ attainment of adequate dialysis in this study is similar to earlier studies, it is worth noting that the insured had a staggering $78.6 \%$ dialysis adequacy compared to a dismay $3.3 \%$ in the uninsured. Somji et $\mathrm{al}^{40}$ in Tanzania, a low medium income nation, found that $40 \%$ of dialysis sessions were adequate, likewise, El-Sheikh et al in Egypt found that $60 \%$ of the dialysis sessions were inadequate. Even higher doses are reported in advanced nations. $^{41,42}$

Renal transplant is associated with even greater challenges to the uninsured. As kidney disease goes through the stages, the cost of treatment increases but at the transplant phase, in addition to higher financial demand, cultural beliefs and biases have limited availability of both live and cadaveric donors. ${ }^{43}$

Some limitation we encountered included our inability to determine participants' dry weight which would have helped us to access its contribution to the delivered dialysis dose. We were unable to determine residual kidney function (RKF). Timing of dialysis was not very regular in some cases. The inclusion of both the predialysis and peridialysis phases of the disease strengthened the study.

\section{CONCLUSION}

Access to renal health care is very low in our clime and expectedly so in many LINs in SSA. Only $11.9 \%$ of the CKD cohorts had health insurance, these were more likely to be males, highly educated, and have lower blood pressure, higher hematocrit, and albumin. The mean dialysis dose for the study was $1.16 \pm 0.4$ with the insured, $1.34 \pm 0.9$ and the uninsured, $1.13 \pm$ 0.5 . (males $1.21 \pm 0.3$, and females $1.07 \pm$ $0.5) .15 .2 \%$ of the sessions were adequate (78.6\% for the insured and $3.3 \%$ for the uninsured). The uninsured had higher anion gap, more metabolic acidosis and were more likely to younger and being the most active working population group, their affectation only further worsens the burden associated with CKD.

\section{Recommendations}

1. There is need for more involvement by the various governments, corporate organizations, religious bodies and philanthropists in developing nations in funding renal care and the removal of bottlenecks against the effective funding of renal health care considering its huge cost.

2. The establishment of a revolving fund for the health sector with emphasis on common chronic and funds consuming diseases, particularly kidney disease, should be set up with contributions from the central and regional governments and multinational organizations.

3. The world Health Organization and other Donor agencies should consider and implement further increases in the funding of health care in low and medium income nations. 
4. Finally, the international community should help the low income nations in rechanneling their funds stalked in foreign banks and similar institutions, by their privileged few, back to these nations to fund health care.

\section{ACKNOWLEDGEMENT}

We appreciate the nurses, laboratory and other supporting staffs of the hospital for their contribution towards the success of this study.

\section{Conflict of Interest: None}

\section{Source of Funding: None}

\section{Ethical Approval: Approved}

\section{REFERENCES}

1. Kaze, A.D., Ilori, T., Jaar, B.G. et al. Burden of chronic kidney disease on the African continent: a systematic review and meta-analysis. BMC Nephrol 19, 125 (2018). https://doi.org/10.1186/s12882-0180930-5

2. World Kidney Day: Chronic Kidney Disease.

2015; http://www.worldkidneyday.org/faqs/ chronic-kidney-disease/ (accessed: Jan 24, 2017).

3. Daar AS, Singer PA, Persad DL, Pramming SK, Matthews DR, Beaglehole R, Bernstein A, Borysiewicz LK, Colagiuri S, Ganguly $\mathrm{N}$, et al. Grand challenges in chronic noncommunicable diseases. Nature. 2007; 450(7169): 494-6.

4. Stevens PE, Levin A. Evaluation and management of chronic kidney disease: synopsis of the kidney disease: improving global outcomes 2012 clinical practice guideline. Ann Intern Med. 2013; 158(11):825-30.

5. Pruijm MT, Madeleine G, Riesen WF, Burnier M, Bovet P. Prevalence of microalbuminuria in the general population of Seychelles and strong association with diabetes and hypertension independent of renal markers. J Hypertens. 2008; 26(5): 871-7.

6. Wen CP, Cheng TY, Tsai MK, Chang YC, Chan HT, Tsai SP, Chiang PH, Hsu CC, Sung PK, Hsu YH, et al. All-cause mortality attributable to chronic kidney disease: a prospective cohort study based on 462293 adults in Taiwan. Lancet (London, England). 2008; 371(9631):2173-82.

7. Ifudu O, Dawood M, Iofel Y, Valcourt JS, Friedman EA. Delayed referral of black, Hispanic, and older patients with chronic renal failure 1999 Am J Kidney Dis 1999; 33(4): 728-33. doi: 10.1016/s02726386(99)70226-x

8. Kidney Disease: Improving Global Outcomes (KDIGO) CKD Work Group. KDIGO 2012 Clinical Practice Guideline for the Evaluation and Management of Chronic Kidney Disease. Kid Int. Suppl. 2013; 3: 1-150.

9. Arije A, Kadiri S \& Akinkugbe OO. The viability of haemodialysis as a treatment option for renal failure in a developing economy. Afr J Med med Sci 2000; 29: 311-314.

10. Akinsola W, Odesanmi WO, Ogunniyi JO, Ladipo GOA. Diseases causing chronic renal failure in Nigerians - a prospective study of 100 cases. Afr J Med Sci. 1989; 18:131-137.

11. Bamgboye E. Haemodialysis: Management problems in developing countries, with Nigeria as a surrogate. Kidney Int 2003; 63 (Suppl 83): S93-S95.

12. Uduagbamen PK, Kadiri S. Intradialysis hypotension and hypertension in patients with end stage kidney disease in Nigeria: risk factors and clinical correlates Ghana Med J 2021; 55(1): 34-42 doi: http://dx.doi.org/10.4314/gmj.v55i1.6

13. Uduagbamen PK, Ajayi SO, Soyinka FO Kadiri S. Impact of Metabolic Acidosis on the Dialysis Dose: Findings from a two center cross-sectional study in a low income population setting Afr J Health Sci 2021; 34(3): 329-341.

14. Dutta A, Hongoro C. Scaling Up National Health Insurance in Nigeria: Learning from Case Studies of India, Colombia, and Thailand. Washington, DC: Futures Group, Health Policy Project. 2013

15. Alebiosu CO, Ayodele OO, Abbas A, Olutoyin AI: Chronic Renal Failure at the Olabisi Onabanjo University Teaching Hospital, Sagamu, Nigeria. Afr Health Sci 2006, 6(3):132-138.

16. National kidney foundation. KDOQI Clinical Practice Guidelines for Diabetes 
and CKD: 2012 update. Am J Kidney Dis 2012; 60(5): 850-886.

17. Traynor JP, Oun HA, McKenzie P, Shilliday LR, Mckay IG, Dunlop A et al. Assessing the utility of the stop dialysate flow method in patients receiving haemodiafiltration. Nephrol Dial Trans 2005; 20(11): 2479-2484

18. Daugirdas JT. Second generation logarithmic estimates of single-pool variable volume $\mathrm{Kt} / \mathrm{V}$ : an analysis of error. J Am Soc Nephrol 1993; 4(5): 1205-13.

19. Clase CM, St Pierre MW, Churchill DN. Conversion between bromocresol greenand bromocresol purple-measured albumin in renal disease Nephrol Dial Trans 2001; 16: 1925-1929

20. Meng L, Yu W, Wang T, Zhang L, Heerdt P, Gelb .AW. (2018) Blood Pressure Targets in Perioperative Care. Provisional Considerations Based on a Comprehensive Literature Review. BMJ Hypertension 72, 806-817

21. Classification and Diagnosis of Diabetes: Standards of Medical Care in Diabetes- 2021ADA Diabetes Care 2021 44(Supplement 1): S15S33.https://doi.org/10.2337/dc21-S002

22. Cappellini MD, Mota I. (2015) Anemia in Clinical Practice-Definition and Classification: Does Hemoglobin Change With Aging? Seminars in Hematology 52, 261-269

https://doi.org/10.1053/j.seminhematol.2015 .07 .006

23. Levey AS, Stevens LA, Schmid CH, Zhang YL, AFr C, Feldman HI, et al. (2009) A new equation to estimate glomerular filtration rate. Ann Intern Med. 150, 60412. https://doi.org/10.7326/0003-4819-1509-200905050-00006

24. Weaving G, Batstone CF, Jones RG. Age and sex variation in serum albumin concentration: an observational study Annal Clin Biochem 2016, 53(1): doi.org/10.1177/0004563215593561

25. Assimon M.M., Wang L, Flythe J.E. Failed target weight Achievement Associates with short-term hospital encounters among individuals receiving maintenance hemodialysis. J. Am. Soc. Nephrol. 2018; 29(8):2178-2188

26. Okoye OC, Slater HE, Rajora N. Prevalence and risk factors of intra-dialytic hypotension: a 5 year retrospective report from a a single Nigeria Centre. Pan Afr Med J 2017; 28(62): 13743

27. Van Buren PN, Kim C, Toto RD, Inrig JK. The prevalence of persistent intradialytic hypertension in a hemodialysis population with extended follow-up. Int $J$ Artif Organs. 2012; 35:1031-1038.

28. Kaze FF, Meto DT, Halle MP, Ngogang J, Kengne AP. Prevalence and determinants of chronic kidney disease in rural and urban Cameroonians: a cross-sectional study. BMC Nephrol. 2015; 16: 117.

29. Luycky VA, Cherney DZI, Bello AK. Preventing CKD in developed countries. Kidney Intl Reports 2019; 6(3): 263-277. Doi org/10.1016/j ekri 201912.003

30. Mallappallil M, Friedman EA, Delano BG, McFarlane SI, Salifu MO. Chronic kidney disease in the elderly: evaluation and management Clin Pract (Lond) 2014; 11(5): 525-535. doi: 10.2217/cpr.14.46

31. Ulasi, C. Ijoma, E. Arodiwe, O. Onodugo and J. Okoye, Lifestyle risk factors and markers of chronic kidney disease: A community based study, A presentation at the AFRAN Congress, Abuja, Nigeria. 2009

32. Unuigbe E.I. Funding renal care in Nigeria: a critical appraisal. Trop J Nephrol. 2006; 1: 33-38.

33. Arogundade FA, Barsoum RS. Chronic kidney disease in sub Saharan African: A call for government, non-government and community support. Am J Kidney Dis 2008; 51: 515-23

34. Miller, J.A., Cherney, D.Z., Duncan, J.A., Lai, V., Burns, K.D., Kennedy, C.R.J et al. Gender Difference in the Renal Response to Renin Angiotensin System Blockage. J Am Soc Nephrol, 2006; 17: 2554-2560. doi.org/10.1681/ASN.2005101095

35. Verzola D, Gandolfo MT, Salvatore F, Villaggio B, Gianiorio F, Traverso P, et al. Testosterone promotes apoptotic damage in human renal tubular cells Kidney Intl 65(4):1252-61.DOI: 10.1111/j.15231755.2004.00497.x

36. Davis E, Campbell K, Gobe G, Hawley $\mathrm{C}$, Isbel N, Johnson DW. Association of anthropometric measures with kidney disease progression and mortality: a retrospective cohort study of pre-dialysis chronic kidney disease patients referred to a specialist renal service BMC Nephrol. 2016; 17: 74. doi: 10.1186/s12882-016-0290-y 
37. Raikou VD, Kyriaki.The Association between Intradialytic Hypertension and Metabolic Disorders in End Stage Renal Disease. Int J Hypertens 2018; https://doi.org/10.1155/2018/1681056

38. Obermayr RP, Temml C, Gutjahr G, Kainz A, Klauser-Braun R, Függer R, et al. Body mass index modifies the risk of cardiovascular death in proteinuric chronic kidney disease. Nephrol Dial Transplant. 2009; 24(8):2421-8. doi: 10.1093/ndt/ gfp075

39. Frassetto LA, Sebastian A, DuBose TD. How metabolic acidosis and kidney disease may accelerate the aging process Eur J Clin Nut 2020; 74, 27-32

40. Somji SS, Ruggajo P, Moledina S. Adequacy of hemodialysis and Its Associated Factors among Patients Undergoing Chronic Hemodialysis in Dares Salam, Tanzania. Int J Nephrol 2020; 10 : 2020:9863065

41. El-Sheikh M, El-Ghazaly G. Assessment of hemodialysis adequacy in patients with chronic kidney disease in the hemodialysis unit at Tanta University Hospital in Egypt. Indian J Nephrol 2016; 26(6): 398-404.

42. Ponte B, Pruijm M, Marques-Vidal P, Martin PY, Burnier M, Paccaud F, et al Determinants and burden of chronic kidney disease in the population-based CoLaus study: a cross-sectional analysis. Nephrol Dial Transplant. 2013 28(9): 2329-39. doi: 10.1093/ndt/gft206. Epub 2013 Jul 3.PMID: 23825103

43. Ajayi SO, Raji Y, Salako BL. Ethical and legal issues in renal transplantation in Nigeria Saudi J Kidney Dis 2016, 27(1): 125-128 DOI: 10.4103/1319-2442.174146

How to cite this article: Uduagbamen PK, Ogunkoya JO, Alalade BA et.al. Chronic kidney disease: socioeconomic impact. findings from a two center study in Southwestern Nigeria. Int $J$ Health Sci Res. 2021; 11(10): 336-347. DOI: https://doi.org/10.52403/ijhsr.20211044 